\title{
Current understanding of rheumatoid arthritis therapy in pregnancy
}

\author{
Sujata Swain ${ }^{1}$, Paresh Jena ${ }^{2}$
}

\author{
${ }^{1}$ Department of Obstetrics and Gynaecology, S.C.B. Medical College, Cuttack, Odisha, India \\ ${ }^{2}$ Department of Rheumatologist, Kalinga Hospital Bhubaneswar, Odisha, India
}

Received: 06 August 2016

Accepted: 06 September 2016

\author{
*Correspondence: \\ Dr. Sujata Swain, \\ E-mail: sjt_swain@yahoo.co.in
}

Copyright: (C) the author(s), publisher and licensee Medip Academy. This is an open-access article distributed under the terms of the Creative Commons Attribution Non-Commercial License, which permits unrestricted non-commercial use, distribution, and reproduction in any medium, provided the original work is properly cited.

\begin{abstract}
Pregnancy in most cases is associated with remission of rheumatoid arthritis (RA), but a quarter of patients continue to have active disease or even worsening of the disease and most patients who improve, relapse in the postpartum period. Patients with RA do not have decreased fertility. A majority of patients with RA may go in remission, but patients who continue with the disease activity require treatment. Drugs that may be safely used during pregnancy include NSAIDs except in third trimester, corticosteroids as low dose prednisone. The preferred disease-modifying agents (DMARDs), during pregnancy are sulfasalazine and hydroxychloroquine. Azathioprine and cyclosporine can be used if the benefits outweigh the risks. Methotrexate and lefunomide are contraindicated and must be prophylactically withdrawn before a planned pregnancy. Biologics like anti-TNF agents, rituximab and abatacept generally should be stopped when pregnancy is discovered. An overall rational approach is highly warranted to treat RA during pregnancy.
\end{abstract}

Keywords: Biologics, DMARDs, Disease activity, Oligohydramnios, Rheumatoid arthritis, Remission, Teratogenic

\section{INTRODUCTION}

Rheumatoid arthritis is a systematic autoimmune disease that leads to chronic inflammation of the joint and other structures. Without use of disease modifying agents, RA often leads to progressive deformities of the hands, feet and larger joints. RA is a female predominant disease, affecting women more than men in a 3:1 ratio.

\section{Fertility in R.A.}

It appears that reduced numbers of children among women with RA may be related to choice rather than to reduced fertility or increased pregnancy loss. ${ }^{1}$ Reduced sexual activities due to functional limitations from the disease are a further, non-biologic cause of lower birth rates due to reduced opportunity for conception. ${ }^{2}$

There has been a suggestion of reduced ovulatory function among women with RA in a small study of menstruating women, but this has yet to be followed up with larger studies. A recent population based study from Norway has found that women with RA used assisted reproduction technologies more than age matched healthy women. ${ }^{3}$ Similar finding were seen in a case-control study of pregnant women in the Netherlands (17.8\%RA patients used assisted reproductive techniques compared with $3 \%$ in healthy women). ${ }^{4}$ Preconception treatment strategies should aim at maximum suppression of disease activity, taking account of possible negative effects of NSAIDs use and higher prednisone doses. ${ }^{5}$

\section{Pregnancy outcomes}

Several recently published population based studies have documented an increased risk of preterm birth $(<37$ weeks' gestational age) and small for gestational age (SGA, $<10^{\text {th }}$ percentile weight of gestational age) among women with RA compared healthy women. 
Two prospective cohort studies of pregnant women with RA compared with healthy pregnant women have demonstrated an inverse association between disease activity during third trimester of pregnancy and birth weight. ${ }^{6}$

\section{Disease outcome in pregnancy}

In reviewing the majority of the data looking at disease activity during pregnancy, it appears that approximately $75 \%$ of patients will have some degree of improvement. This may occur as early as the first trimester and continue through the pregnancy. 7,8

De Man et al looked prospectively at 84 patients with RA and determined improvement and deterioration by the DAS28 changes. The women who started pregnancy with moderate disease activity only $48 \%$ had a moderate improvement response. Those with low disease activity during the first trimester tended to remain stable. Only $27 \%$ of the patients as a whole were in remission by the third trimester. ${ }^{9}$

The combined effect of increased levels of cortisol, oestrogen and vitamin D has been implicated in lowering the pro-inflammatory cytokines, IL-12 and TNF- $\alpha$, during pregnancy. ${ }^{10}$

Pregnancy is a situation of induced immunological tolerance in the mother against the semi-allogeneic fetus. Immunological changes in pregnancy necessary for this so-called feto-maternal tolerance are summarized in Fig. 1 and include thymic involution, decreased NK-cell function, and a decrease in Th1 immune response shifting towards a more Th2-dominated immune response pattern, whereas $\mathrm{T}$ - and B-cell numbers, antibody production and response to vaccines do not change during pregnancy. ${ }^{11-15}$

The majority of studies have confirmed that a higher degree of maternal-fetal HLA mismatch is associated with improved disease activity during pregnancy; however a study of 110 maternal-fetal pairs failed to find such an association. More recently a prospective study of 118 pregnant women with RA from the Netherlands found that women who were seronegative for rheumatoid factor or anti-cyclic citrullinated peptide had a greater likelihood of improvement in disease activity during pregnancy. This has yet to be studied in other populations.

Despite a wide range of disease activity during pregnancy, the majority of studies have shown that RA may worsen or flare in the postpartum period. ${ }^{7,9}$ Possible causes for flare-ups during the postpartum period include a decrease in the anti-inflammatory steroid levels,elevated levels of prolactin (ie, proinflammatory hormone), change in the neuroendocrine axis, change from a TH2 to a helper T-cell 1 cytokine profile.-16

\section{Ways to improve outcomes}

Monitoring the disease activity during pregnancy can be tricky because distinguishing underlying activity of RA from normal changes during pregnancy is often not clear. Both erythrocytesedimentation rate (ESR) and C-reactive protein (CRP) are makers of systematic inflammation that are often used to follow disease activity in rheumatic disease. Unfortunately, both these levels can fluctuate in pregnancy with more dramatic increase seen in ESR rather than CRP. ${ }^{9}$

Furthermore, clinical changes of pregnancy such as fatigue, swelling (especially of the distal extremities), and carpal tunnel syndrome are commonly seen in pregnancy. Careful examination of the wrist and assessment of neurologic symptoms are important to distinguish carpel tunnel syndrome from RA flare.

\section{MEDICATION DURING PREGNANCY}

There are three general classes of drugs commonly used in the treatment of rheumatoid arthritis: non-steroidal anti-inflammatory agents (NSAIDs), corticosteroids, and disease modifying anti-rheumatic drugs (DMARDs).

In the ideal situation, women with RA considering pregnancy should aim to achieve good control of disease after discontinuation of medication with teratogenic potential, using medication consider safer during pregnancy if necessary to reduce inflammation.

Certain medications are strictly contraindicated during pregnancy and should always be avoided due to teratogenic potential; methotrexate and leflunomide.

If a disease-modifying agent is required to control chronically active disease during an anticipated or confirmed pregnancy, plaquenil, sulfasalazine (SSZ), and/or azathioprine carry the fewest risks to developing fetus. ${ }^{17}$

\section{Nonsteroidal anti-inflammatory drugs}

Possible effects on the mother include prolonged gestation and labor, increased peripartum blood loss, and increased anaemia. The potential adverse effects to the fetus include impaired fetal renal function with oligohydramnios and increased cutaneous and intracranial bleeding. Monitoring for oligohydramnios should be considered if the pregnant patient is on prolonged NSAID therapy. NSAIDs are contraindicated in the third trimester, because they promote premature closure of the ductus arteriosus, leading to fetal pulmonary hypertension. Ductal constriction can occur at any gestational age; however, one study noted a dramatic increase in indomethacin-induced ductal constriction at 31 weeks' gestation. ${ }^{18}$ 


\section{Corticosteroids}

Corticosteroids are potent anti-inflammatory agents. They are considered relatively safe in pregnancy when used in low doses and are designated as category B medications. If steroid treatment is desired for the mother, hydrocortisone, cortisone, or prednisone should be chosen. For acute flares of disease use of glucocorticosteroids is the best option because they have a rapid onset of action and can be citrated to the level of disease activity. There is vast experience with glucocorticoids during pregnancy for many indicators. Prednisone is perhaps the most ideal choice for the treatment of maternal disease because a very small amount of active drug will enter the fetal circulation. ${ }^{17}$ The lowest possible steroid dose needed to control disease activity should be used in pregnancy. Stress doses of steroids should be used during labor and delivery if the mother received steroids (even low-dose) for more than 2-3 weeks during pregnancy, and the neonate should be monitored for evidence of adrenal insufficiency and infection.

\section{DISEASE MODIFYING ANTI-RHEUMATIC DRUGS (DMARDS)}

\section{Methotrexate}

Methotrexate, a folic acid antagonist, is contraindicated in pregnancy (category $\mathrm{X}$ ), because it is an abortifacient and has teratogenic effects, such as causing the development of craniofacial abnormalities, limb defects, and such CNS defects as anencephaly, hydrocephaly, and meningo myelopathy, especially with first-trimester exposure. ${ }^{19}$ Because its active metabolites have a long half-life, methotrexate must be discontinued at least 3 months before conception; compensatory treatment with folic acid should be continued during that period and throughout pregnancy. Male partners should also discontinue methotrexate at least 3 months prior to attempting to conceive.

\section{Leflunomide}

Leflunomide, a pyrimidine synthesis inhibitor, is also a category X medication; it is extremely teratogenic and is absolutely contraindicated in pregnancy. Additionally these agents should be discontinued immediately on the discovery of pregnancy in a woman receiving these medication (leflunomide will require an additional washout with cholestyramine).$^{20}$

\section{Sulfasalazine}

Sulfasalazine (SSZ), a dihydrofolate reductase inhibitor, is a category B medication; it does not increase fetal morbidity or mortality and is considered safe in pregnancy. ${ }^{21}$ If a disease modifying agent is required to control chronically active disease during an anticipated or confirmed pregnancy, plaquenil, sulfasalazine (SSZ), and/or azathioprine carry the fewest risk to the developing fetus and are generally considered relatively safe. $^{17}$

\section{Hydroxychloroquine}

$\mathrm{HCQ}$, an antimalarial agent, is considered a category $\mathrm{C}$ medication. Previous reports of fetal toxicity with this agent were based on the effects of chloroquine, which has 2.5 times the amount of tissue deposition as HCQ. No fetal toxicity is associated with HCQ at the dosage used for RA and connective-tissue disease $(6.5 \mathrm{mg} / \mathrm{kg}$ body weight). ${ }^{22}$ Several studies and case series have provided further evidence that no fetal toxicity is associated with HCQ therapy in mothers. ${ }^{23-25}$

\section{BIOLOGICS}

\section{Tumor necrosis factor-alpha antagonists}

The use or continuation of antitumor necrosis factor (TNF) agents during pregnancy has been more controversial.Medications in the anti-tumor necrosis factor (TNF)-alpha class (e.g. etanercept, adalimumab, infliximab) are commonly used in the treatment of RA. These agents have been labeled as class B medications; animal studies have shown no harm to the fetus but thus far, no randomized, blinded, placebo-controlled trials on potential teratogenicity in humans have been completed. $^{26,27}$ Some studies have suggested an increased risk for congenital abnormalities, whereas others have not. ${ }^{28}$ In case of severe inflammatory disease before or during pregnancy, TNF inhibitors may be used with caution, provided that the mother is aware of and comfortable with the risk to benefit the ratio.

\section{B-Cell depletion}

Rituximab (rituxan), a monoclonal antibody that inhibits CD20 antigen on B lymphocytes, is currently a pregnancy category $\mathrm{C}$ medication. Rituximab is indicated for the treatment for moderate to severe RA. Case reports have also shown that rituximab therapy results in detectable levels of the drug in cord blood and results in B-cell depletion in the mother and the neonate. ${ }^{29}$

\section{Anakinra}

Anakinra (kineret), an interleukin 1 receptor antagonist, is used to treat severe RA. No studies or case reports on the use of this medication during human pregnancy or lactation were found in the literature or reported in data provided by the drug manufacturer. Anakinra is a pregnancy category $\mathrm{B}$ medication. Ostensen et al recommend in a 2011 report that because of the very limited experience with anakinra in pregnancy, its use cannot be recommended. They confirm again that no controlled studies have been published in 2009-2010 on the use of anakinra in pregnancy. ${ }^{30}$ 


\begin{abstract}
Abatacept
Abatacept (orencia) is a selective costimulation modulator that binds to CD80 and CD86, thereby inhibiting activation of $\mathrm{T}$ lymphocytes and interactions with CD28. This drug is indicated for the treatment of moderate to severe RA. ${ }^{31}$ Abatacept is a pregnancy category $\mathrm{C}$ medication; it has been found to cross the placenta and is excreted in rat milk.
\end{abstract}

\section{Medication during postpartum and breastfeeding}

NSAIDs can be used with caution, provided newborns do not have jaundice, because NSAIDs can displace bilirubin and predispose patients to kernicterus. If NSAIDs must be used during breastfeeding, only NSAIDs with a known safety record should be used. ${ }^{19}$ Prednisone can be used safely during breastfeeding, because only small amounts (5\% of the glucocorticoid dose) are secreted in breast milk. Hydroxychloroquine HCQ can potentially displace bilirubin and result in the development of kernicterus. The drug should be discontinued if the neonate has jaundice. Methotrexate is excreted in breast milk in low concentrations and can accumulate in neonatal tissues; thus, it is contraindicated during breastfeeding. ${ }^{32}$ Leflunomide is contraindicated during breastfeeding. Breastfeeding is contraindicated during azathioprine (AZP) therapy because the drug is transferred into maternal milk. ${ }^{33}$ Sulfasalazine (SSZ) is believed to be safe during nursing, although the American academy of pediatrics has cited a reported case of bloody diarrhea in an infant as a reason to use caution with this drug during nursing. ${ }^{34}$ A prospective study in 3 nursing mothers treated with infliximab for Crohn disease found that infliximab was not detectable in their breast milk or in their infants' sera. ${ }^{35}$ Because no data are available on the use of TNF-alpha antagonists, anakinra, abatacept, rituximab in breastfeeding, lactation should be avoided during therapy with these agents. ${ }^{19}$

\section{CONCLUSION}

There are a wide variety of medications available to treat patients with rheumatoid arthritis, many of which are considered unsafe during pregnancy. It is important to tailor a treatment regimen that stabilises the woman's disease prior to conception, using medications that are safe to continue throughout pregnancy and the postpartum period. Drugs that may be safely used during pregnancy include NSAIDs, corticosteroids, plus several DMARDs, including sulfasalazine and hydroxychloroquine. Drugs recommended to be stopped before pregnancy includes methotrexate and leflunomide, plus the biologics: anti-TNF agents, rituximab and abatacept. In this review, we provide an overview of the RA treatment issues pre-conception, during pregnancy and in the post-partum period with respect to breastfeeding, and we provide guidelines for drugs that may be used relatively safely for RA management in pregnant women.
Funding: No funding sources

Conflict of interest: None declared

Ethical approval: Not required

\section{REFERENCES}

1. Katz PP. Childbearing decisions and family size among women with rheumatoid arthritis. Arthritis Rheu. 2006;54:899-907.

2. Kraaimaat FW, Bakker AH, Janssen E, Bijlsma JW. Intrusiveness of rheumatoid arthritis on sexuality in male and female patients living with a spouse. Arthritis Care Res. 1996;9(2):120-5.

3. Flaiser F, Heden B, Sany J, Combe B. A study of ovarian function in rheumatoid arthritis. Rev Rheum. 1995;62:549-54.

4. Wallenius M, Skomsvoll JE, Irgens LM. Pregnancy and delivery in women with chronic inflammatory arthritis with specific focus on birth order. Arthritis Rheum. 2011;60:1534-42.

5. Man YA, Hazes JMW, Heide VH. Association of higher rheumatoid arthritis disease activity during pregnancy with lower birth weight. Arthritis Rheum. 2009;60:3196-206.

6. Bowden AP, Barrett JH, Fallow W, Silman AJ. Women with inflammatory poly arthritis have babies of lower birth weight. J Rheumatology. 2001;28:3559.

7. Ostensen M, Aune B, Husby G. Effect of pregnancy and hormonal changes on the activity of rheumatoid arthritis. Scand J Rheumatology. 1983;12:69-72.

8. Nelson JL, Hughes KA, Smith AG. Maternal and fetal disparity in HLA class II alloantigens and the pregnancy induced ameloriation of rheumatoid arthritis. N Engl J Med. 1993;329:466-71.

9. Man YA, Dolhain RJ, Geijn FE. Disease activity of rheumatoid arthritis during pregnancy results from nation-wide prospective study. Arthritis Rheum. 2008;59:1241-8.

10. Elenkov IJ, Wilder RL, Bakalov VK, et al. IL-12, TNF-alpha, and hormonal changes during late pregnancy and early postpartum: implications for autoimmune disease activity during these times. J Clin Endocrinol Metab. 2001;86:4933-8.

11. Tibbetts TA, DeMayo F, Rich S, Conneely OM, Malley BW. Progesterone receptors in the thymus are required for thymic involution during pregnancy and for normal fertility. Proc Natl Acad Sci USA. 1999;96:12021-6.

12. Rango U. Fetal tolerance in human pregnancy a crucial balance between acceptance and limitation of trophoblast invasion. Immunol Lett. 2008;115:21-32.

13. Forger F, Marcoli N, Gadola S, Moller B, Villiger PM, Ostensen M. Pregnancy induces numerical and functional changes of CD4+CD25 high regulatory $\mathrm{T}$ cells in patients with rheumatoid arthritis. Ann Rheum Dis. 2008;67:984-90.

14. Kahn DA, Baltimore D. Pregnancy induces a fetal antigen-specific maternal $\mathrm{T}$ regulatory cell response 
that contributes to tolerance. Proc Natl Acad Sci. 2010;107:9299-304.

15. Whitacre CC. A gender gap in autoimmunity. Science. 1999;283:1277-8.

16. Zoli A, Lizzio MM, Ferlisi EM, Massafra V, Mirone $\mathrm{L}$, Barini A, et al. ACTH, cortisol and prolactin in active rheumatoid arthritis. Clin Rheumatol. 2002;21(4):289-93.

17. Ostenen M, Lockshin M, Doria A. Update on safety during pregnancy of biological agents and some immunosuppressive anti-rheumatic drugs. Rheumatology. 2008;47(3):28-31.

18. 18. Vermillion ST, Scardo JA, Lashus AG, Wiles HB. The effect of indomethacin tocolysis on fetal ductus arteriosus constriction with advancing gestational age. Am J Obstet Gynecol. 1997;177(2):256-9.

19. Temprano KK, Bandlamudi R, Moore TL. Antirheumatic drugs in pregnancy and lactation. Semin Arthritis Rheum. 2005;35(2):112-21.

20. Chambers CD, Johnson DL, Robinson LK. Organisation of teratology information specialists collaborative research group birth outcomes in women who have taken leflunomide during pregnancy. Arthritis Rheum. 2010;62:1494-503.

21. Mogadam M, Dobbins WO, Korelitz BI, Ahmed SW. Pregnancy in inflammatory bowel disease: effect of sulfasalazine and corticosteroids on fetal outcome. Gastroenterology. 1981;80(1):72-6.

22. Parke A, West B. Hydroxychloroquine in pregnant patients with systemic lupus erythematosus. J Rheumatol. 1996;23(10):1715-8.

23. Buchanan NM, Toubi E, Khamashta MA, Lima F, Kerslake S, Hughes GR. Hydroxychloroquine and lupus pregnancy: review of a series of 36 cases. Ann Rheum Dis. 1996;55(7):486-8.

24. Costedoat CN, Amoura Z, Duhaut P, Huong DL, Sebbough D, Wechsler B, et al. Safety of hydroxychloroquine in pregnant patients with connective tissue diseases: a study of one hundred thirty-three cases compared with a control group. Arthritis Rheum. 2003;48(11):3207-11.

25. Motta M, Tincani A, Faden D, Zinzini E, Chirico G. Antimalarial agents in pregnancy. Lancet. 2002;359(9305):524-5.

26. Barrett JH, Brennan P, Fiddler M, Silman AJ. Does rheumatoid arthritis remit during pregnancy and relapse postpartum? results from a nationwide study in the United Kingdom performed prospectively from late pregnancy. Arthritis Rheum. 1999;42(6):1219-27.

27. Treacy G. Using an analogous monoclonal antibody to evaluate the reproductive and chronic toxicity potential for a humanized anti-TNFalpha monoclonal antibody. Hum Exp Toxicol. 2000;19(4):226-8.

28. Clowse ME. The use of anti TNF $\alpha$ medications for rheumatologic disease in pregnancy. Int $\mathrm{J}$ Women Health. 2010;2:199-209.

29. Klink DT, Elburg RM, Schreurs MW, Well GT. Rituximab administration in third trimester of pregnancy suppresses neonatal B-cell development. Clin Dev Immunol. 2008;2008:271363.

30. Ostensen M, Forger F. Treatment with biologics of pregnant patients with rheumatic diseases. Curr Opin Rheumatol. 2011;23(3):293-8.

31. Use of orencia during pregnancy and effects on fertility, personal communication with Kelly S. Park, PharmD, MBA, representative of Bristol-Myers Squibb.: Bristol-Myers Squibb. 2008.

32. Johns DG, Rutherford LD, Leighton PC, Vogel CL. Secretion of methotrexate into human milk. Am J Obstet Gynecol. 1972;112(7):978-80.

33. EBPG expert group on renal transplantation. European best practice guidelines for renal transplantation. Long-term management of the transplant recipient. Pregnancy in renal transplant recipients. Nephrol Dial Transplant. 2002;17(4):505.

34. American Academy of Pediatrics. The transfer of drugs and other chemicals into human milk. Pediatrics. 1994;93(1):137-50.

35. Kane S, Ford J, Cohen R, Wagner C. Absence of infliximab in infants and breast milk from nursing mothers receiving therapy for Crohn's disease before and after delivery. J Clin Gastroenterol. 2009;43(7):613-6.

Cite this article as: Swain S, Jena P. Current understanding of rheumatoid arthritis therapy in pregnancy. Int J Reprod Contracept Obstet Gynecol 2016;5:3275-9. 